(ABR), dichotic digit test (DD). We examined auditory fatigue through the EchoScan test.

Results Exposed workers had worse thresholds bilaterally at 4 $\mathrm{kHz}(\mathrm{p}=0.0253$ in right ear - RE; $\mathrm{p}=0.0436$ in left ear LE). Ipsilateral acoustic reflex levels were different between groups at the frequencies of $500 \mathrm{~Hz}(p=0.02$ in RE; $p=0.04$ in LE) and $1 \mathrm{kHz}(\mathrm{p}=0.04$ in LE). The only differences detected by the $\mathrm{ABR}$ was for the interpeak interval III-V $(p=0.03$ in RE; $p=0.02$ in LE). DD means (exposed $=89.5$ \pm 13.33 ; control $=96.40 \pm 4.46$ ), were only different in the LE $(p=0.05)$. Echoscan detected a difference $(p=0.0317)$ between the means of the exposed $(4.58 \pm 6.8)$ and control $(0 \pm 4.62)$ groups, indicating possible hearing fatigue in the exposed group.

Conclusion Each of the hearing tests indicated differences between the groups, suggesting that occupational co-exposure to low noise and low concentrations of solvents can be harmful to hearing functions. The temporary effect of the exposure was detected by the EchoScan and the acoustic reflex of the stapedius muscle. The permanent effects were identified mainly at the level of the high brainstem and in the auditory ability of binaural integration. The EchoScan, can facilitate the early identification of auditory effects, while these are still temporary.

\section{P-178 EXPOSURE-RESPONSE RELATIONSHIPS FOR WOOD DUST EXPOSURE AND WORK-RELATED ASTHMA IN MOZAMBIQUAN WOOD PROCESSING WORKERS}

${ }^{1}$ Paulino Chamba, Roslynn Baatjies, Mohamed Jeebhay. 'Centre of Medical Examinations, Mozambique

\subsection{6/OEM-2021-EPI.221}

Introduction While exposure-response relationships for workrelated asthma have been studied in wood processing workers, few studies have used multiple exposure indices to evaluate various clinical endpoints.

Objectives To investigate exposure-response relationships for work-related respiratory symptoms, airway obstruction, bronchial hyperresponsiveness, and exhaled nitric oxide (FeNO) among Mozambiquan wood processing workers.

Methods A cross-sectional study of 450 workers using inhalable wood dust particulate concentrations assigned to workers based on personal environmental exposure samples $(n=124)$ collected for different jobs. Exposure metrics of current and cumulative dust exposure were modeled using multivariate linear and logistic regression for the clinical endpoints, adjusted for confounders (age, gender, atopy, smoking).

Results The mean inhalable dust concentrations modelled were $3.29 \mathrm{mg} / \mathrm{m} 3$ (GSD: 3.04), cumulative exposure in current job was $26.23 \mathrm{mg} / \mathrm{m} 3-y r$ (GSD: 2.94) and cumulative exposure over all jobs was $24.07 \mathrm{mg} / \mathrm{m} 3-\mathrm{yr}$ (GSD: 2.94). The mean age of predominately male workers was 38 years, among whom $76 \%$ were non-smokers and 50\% atopic. Work-related ocularnasal symptoms were associated, in a dose-dependent manner, with exposure groups (EGs) categorized according to current dust particulate levels (medium, 4.68-4.71 mg/m3: OR = 3.45, 95\% CI: 1.21 - 10.63; high, >4.71 mg/m3: OR = 3.66, 95\% CI: 1.19 - 11.26) versus low EG ( $<4.68 \mathrm{mg} /$ $\mathrm{m} 3)$ in adjusted models. Work-related asthma symptoms were associated with mean cumulative exposure $(\mathrm{OR}=1.01,95 \%$ CI: $1.00-1.02)$ in adjusted models. FeNO was associated with cumulative exposure in current job (quartile 2, OR= 1.23, 95\% CI: $1.02-1.51$; quartile $4, \mathrm{OR}=1.23,95 \% \mathrm{CI}$ : $1.02-1.49$ ) as well cumulative exposure across all jobs in the factory (quartile $4, \mathrm{OR}=1.22,95 \% \mathrm{CI}: 1.01-1.48$ ) versus quartile 1 in adjusted models.

Conclusion This study demonstrated positive exposure-response relationships between wood dust particulate for both workrelated ocular-nasal symptoms as well as asthma, suggestive of an acute irritant.

\section{P-180 COHORT PROFILE: THE DANISH OCCUPATIONAL MEDICINE COHORT - LONG-TERM PROGNOSIS FOR PATIENTS WITH WORK-RELATED DISORDERS}

${ }^{1}$ Vita Ligaya Dalgaard, Morten Vejs Willert, Marianne Kyndi, Jesper Medom Vestergaard, Johan Hviid Andersen, David Høyrup Christiansen. 'Aarhus BSS, Aarhus University Council, Denmark

\subsection{6/OEM-2021-EPI.222}

Objective Danish departments of occupational medicine currently receive about 9,000 patients each year referred due to suspected work-related disorders. In Denmark and internationally there is a lack of follow-up studies investigating the longterm prognosis of employees with work-related disorders. The purpose of this cohort is to facilitate long-term prognostic studies.

Methods The cohort was created through The Danish National Patient Register and comprises all patients seen in Danish Departments/units of Occupational Medicine from 2000-2018 $(\mathrm{N}=145,390)$. Numerous register data are included in the database from five years prior to time of inclusion until 2018. Examples of register data are; information on work, sickness absence and disability as well as number and type of contacts to health services, hospital admissions, income, education, type of occupation, social status, death and cause of death. Job Exposure Matrices (JEMs) on physical and psychosocial work exposure, life styles and other matrices are also available. The cohort will be updated with new patients regularly.

Results Currently the cohort comprises seven major groups: musculoskeletal $(\mathrm{n}=51.056)$, mental health $(\mathrm{n}=28.212)$, lung $(\mathrm{N}=12.274)$, skin $(\mathrm{N}=6.544)$, nervous system $(\mathrm{N}=5.513)$, cancer $(\mathrm{N}=1.566)$, and others $(\mathrm{n}=40.225)$. Preliminary analyses across groups suggest that the labor market attachment has been high among the majority (about 75\%) of patients during the years prior to the year of inclusion. However, results indicate that the main diagnostic groups, with the exception of skin patients, do not return to comparable levels of labor market attachment during the years following referral and diagnosis.

Conclusions The Danish Occupational Medicine Cohort is an open source dataset available to researchers interested in longterm follow-up on patients with work-related disorders. Preliminary analyses indicate most diagnostic groups do not return to prior levels of labor market attachment during the years following referral and diagnosis from a department of occupational medicine. 